\title{
ELECTROCHEMICAL REDUCTION OF URIDINE IN DIMETHYL SULFOXIDE: EFFECT OF THE RIBOSE GROUP
}

\author{
William T. Bresnahan, Timothy E. Cummings* and Philip J. Elving \\ University of Michigan, Ann Arbor, MI 48109, U.S.A.
}

(Received 21 July 1980)

\begin{abstract}
A batract-The easier electrochemical reduction of uridine (1- $\beta$-D-ribofuranosyluracil) in dimethyl sulfoxide as compared to uracil $(2,4$-dihydroxypyrimidine) by ca. $0.1 \mathrm{~V}$ is explicable on the basis of the electronwithdrawing effect of the ribose group. This effect and possible steric hindrance by the ribose group markedly affect the reaction sequence following the initial one-electron reduction to generate a radical anion, which abstracts a proton from the parent uridine (father-son reaction) to form the neutral uridine free radical and the uridine anion. With increasing uridine concentration, further reduction and protonation reactions are fuvored, resulting in an increase in the effective faradaic $n$ from ca. 0.5 to 0.8 . The availability of only one proton-donating site on uridine, ie, that on $\mathrm{N}(3)$, allows explication of the behavior of other hydroxypyrimidines such as uracil.
\end{abstract}

\section{INTRODUCTION}

The authors and their collaborators have been investigating the electrochemical and related chemical behavior in aqueous and nonaqueous media of the nucleic acid bases, their ribose and ribosophosphate derivatives, and relevant model compounds; one aim is a more detailed understanding of substituent effects on pyrimidine ring reduction. In this connection, a systematic study is being made of the 2-hydroxypyrimidines since all three of the major pyrimidine bases, which occur in nucleic acids, are such compounds; cytosine (4-amino-2-hydroxypyrimidine) is one of the two principal pyrimidines in both DNA and RNA; the other is uracil (2,4-dihydroxypyrimidine) in RNA and thymine (5-methyl-2,4-dihydroxypyrimidine) in DNA.

The electrochemical reduction of 2-hydroxypyrimidine (2-HP) [1], uracil[2] and thymine[3] in dimethyl sulfoxide (DMSO) have been recently described. Reduction of these compounds, $R H$, involves an initial one-electron (1e) addition to generate a radical anion, RH $^{-}$, which abstracts a proton from the unreduced parent pyrimidine to form the neutral free radical, $\mathbf{R H}_{2}$, and the parent compound's conjugate base, $\mathbf{R}^{-}$, (father-son reaction) [4]:

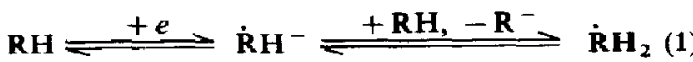

The follow-up father-son reaction of (1) is competitive with other possible follow-up reactions such as dimerization of the radical anion; it would be assisted by further reaction of the free radical, $\mathbf{R H}_{2}$. Other possible reactions include free radical dimerization, radical anion disproportionation, protonation of dimer dianion, $(\mathbf{R H})_{2}^{2-}$, and/or free radical, reduction of the free radical (possible ECE reaction sequence) and protonation of the resulting carbanion; where RH serves as a proton source, non-reducible anion $\mathbf{R}^{-}$is

- Present address: Department of Chemistry, University of Miami, Coral Gables, FL 33125, U.S.A. produced. The relative rates of formation of the various products depend on the original RH concentration and experimental factors such as the time scale of electrolysis as well as the structure of RH[1-4].

The present paper describes the reduction of uridine (1- $\beta$-D-ribofuranosyluracil), the nucleoside of uracil. Since uracil is electrochemically reducible in DMSO as a result of the extended negative potential range uvailable, uridine was investigated in the same solvent.

Electrochemical reduction of uridine is of interest because of the additional information which it might yield on the reduction of other hydroxypyrimidines, $e g$, in respect to effects specific to the proton on N(l) of uracil, which proton is not available in uridine for protonation of the initially generated radical anion ( $c f$. (7) for ring numbering), and to steric influence of the ribose substituent. Uridine provides the initial transition to the ribosophosphate derivatives, in which uracil and the other hydroxypyrimidines exist in nature. In addition to RNA, uracil nucleotides are involved in energy-transfer reactions, eg, uridine-5'diphosphate is the main carrier of sugar residues, particularly as UDP-D-glucose, in the biosynthesis of polysaccharides or energy storage polymers, eg, glycogen, in higher animals[5].

\section{EXPERIMENTAL}

Unless otherwise indicated, experimental details and procedures were the same as those previously described $[1-3]$.

Reagents and solutions. DMSO (Fisher Scientific) was purified by fractional crystallization at $14^{\circ} \mathrm{C}$. Tetra-n-butylammonium perchlorate (TBAP) (G. Frederick Smith Chemical) was dried at $60^{\circ} \mathrm{C}$ under vacuum for $48 \mathrm{~h}$. Tetraethylammonium hydroxide (TEAH; $20 \%$ solution in water) was obtained from Aldrich, and uridine (A grade) from Calbiochem. The solvent system was $0.100 \mathrm{M}$ TBAP in DMSO. Prepurified nitrogen was bubbled through the test sol- 
ution for $30 \mathrm{~min}$. prior to and passed over the solution during electrolysis.

Instrumentation. Electrochemical measurements were made with an in-house-designed rapid responsive potentiostat [6] or a conventional design instrument constructed from operational amplifiers. Cyclic voltammetric potential functions were generated with a Wavetek Model 112 triggered voltage controlled generator. A PAR 121 lock-in amplifier/phase detector was used for ac polarography. Polarograms were recorded with a Houston $2000 x$-y recorder. A Tektronix $5103 \mathrm{~N}$ power supply/amplifier was used with a C-5A camera as the basic oscilloscope system; plug-in amplifiers (5A18N dual trace and 5A15N) were used for $x-y$ measurements. Potentials were displayed with a Heathkit IM-102 digital multimeter or a Hewlett Packard 3440A/3443A digital voltmeter.

Water-jacketed one- and three-compartment cells, maintained at $25^{\circ} \mathrm{C}$, were used; a Luggin capillary was used with the former. The auxiliary electrode was a Pt wire, immersed in background solution. The reference electrode was a modified aqueous saturated calomel electrode[1], against which all potentials are cited. For cyclic voltammetry, a Metrohm E-410 microfeeder was the working electrode.

\section{RESULTS AND DISCUSSION}

Uridine is reduced photochemically in the presence of bisulfite[7] and sodium borohydride[8] to the dihydro derivative. A symmetric dimer bonded at $\mathbf{C}(5)$ and $C(6)$ has been prepared photochemically[9].

In aqueous solution (0.5 M NaF; $0.1 \mathrm{M}$ phosphate buffer, pH 7.10), the ac polarogram of uridine indicated adsorption on the $\mathrm{Hg}$ electrode with a response minimum at $-0.4 \mathrm{~V}$ and a desorption maximum at $-1.0 \mathrm{~V}$; no reduction was observed prior to background discharge at $-1.7 \mathrm{~V}[10,11]$. Unti] the present, electrochemical reduction of uridine has not been reported.

dc Polarography. A typical $d c$ polarogram for the reduction of uridine is given in Fig. 1; the absence of a well-defined plateau increases the uncertainty in the wave parameters (Table 1). The small wave at $-2.0 \mathrm{~V}$, which is due to an impurity in the DMSO $(0.1 \mathrm{M}$
TBAP) solution, may involve the $\mathrm{Na}(\mathrm{I})-\mathrm{Na}(\mathrm{O})$ couple[1].

In the concentration range studied $(0.2$ to $2 \mathrm{mM})$, the diffusion current constant, $I_{d}$, increases and the half-wave potential, $E_{1 / 2}$, bocomes slightly more negative with inceasing concentration (Table 1), which suggests that the mechanism involves one or more chemical reactions. For uracil in DMSO, $I_{d}$ decreased and $E_{1 / 2}$ became more negative with increasing concentration [2]. An $i_{d}-c$ plot for $2-H P$ was linear up to at least $3 \mathrm{mM}$ and $E_{1 / 2}$ became more positive with increasing concentration [1].

When an equivalent molar amount of TEAH is added to a $1.94 \mathrm{mM}$ uridine solution in DMSO, the uridine (RH) reduction wave (2) disappears and an anodic wave appears at $-0.25 \mathrm{~V}$ (Table 1); the latter is due to oxidation of $\mathrm{Hg}$ to $\mathrm{HgR}$ (4) in the presence of uridine anion ( $\mathbf{R}^{-}$), which is formed by reaction of $\mathbf{R H}$ with $\mathrm{OH}^{-}$from TEAH (3).

$$
\begin{aligned}
\mathbf{R H}+e & =\dot{\mathbf{R}} \mathbf{H}^{-} \\
\mathbf{R H}+\mathbf{O H} & =\mathbf{R}^{-}+\mathbf{H}_{2} \mathbf{O} \\
\mathbf{R}^{-}+\mathbf{H g} & =\mathbf{H g R}+e
\end{aligned}
$$

Uridine would be deprotonated at $\mathbf{N}(3)$ to form $\mathbf{R}^{-}$, which is not reducible within the available potential range.

The $I_{d}$ of 0.95 for the uridine anion indicates the pronounced effect of the ribose group in decreasing depolarizer mobility in DMSO; $I_{\mathrm{d}}$ for the uracil anion is 1.33 [2]. If an analogous proportional effect occurs with the parent compounds, the $I_{d}$ of 1.29 expected for a one-electron (1e) reduction of uracil[2] would correspond to an $\boldsymbol{I}_{d}$ of 0.92 for a similar uridine reduction. The latter value would indicate that, at the lowest uridine concentration in Table $1(0.19 \mathrm{mM})$, approximately 58 per cent of the uridine is reduced to the radical anion which deprotonates the remainder of the uridine to produce electroinactive $\mathbf{R}^{-}$.

A plot of $I_{d} v s$ uridine concentration (Table 1) is linear up to $0.6 \mathrm{mM}$ and extrapolates to an $I_{d}$ of $0.43 \mathrm{at}$ zero concentration. The increase in $L$ with concentration may be due to a variety of causes, $e g$, an increase in the fraction of the free radical $\left(\dot{R} \mathbf{H}_{2}\right)$ reduced (perhaps in an FC.F reaction sequence), a decrease in the fraction of RH which serves as protonation agent, a combination of these and other effects, eg, the radical anion disproportionation subsequently discussed. A

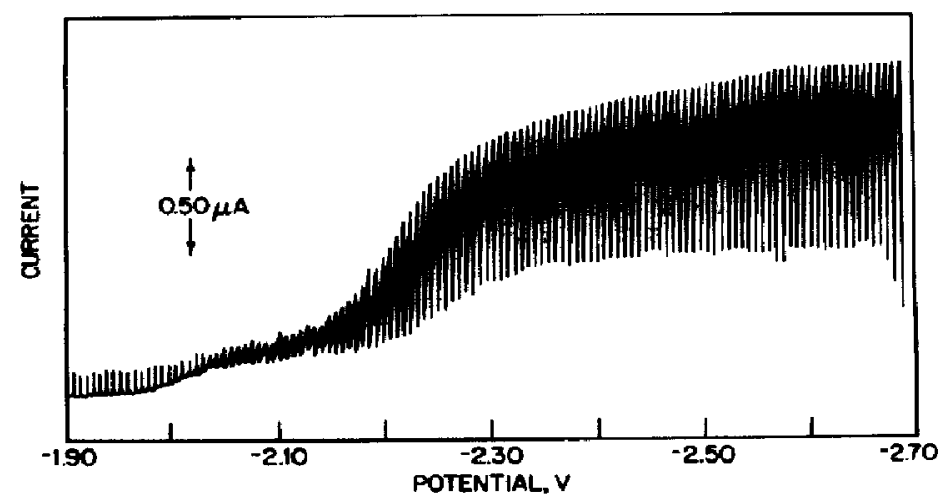

Fig. 1. d.c. polarogram of $1.94 \mathrm{mM}$ uridine in DMSO (0.1 M TBAP). 
Table 1. de Polarographic reduction of uridine in DMSO $(0,1 \mathrm{M} \text { TBAP })^{*}$

\begin{tabular}{lccc}
\hline $\begin{array}{c}\text { Concn } \\
(\mathrm{mM})\end{array}$ & $I_{\mathrm{d}}{ }^{+}$ & $\begin{array}{c}-E_{1 / 2} \\
(\mathrm{~V})\end{array}$ & $\begin{array}{c}E_{1 / 4}-E_{3 / 4} \\
(\mathrm{mV})\end{array}$ \\
\hline 0.190 & 0.53 & 2.20 & 53 \\
0.455 & 0.61 & 2.23 & 62 \\
0.569 & 0.69 & 2.20 & 65 \\
0.582 & 0.70 & 2.21 & 53 \\
1.94 & 0.73 & 2.22 & 70 \\
1.94 & 0.76 & 2.22 & 67 \\
1.94 & 0.95 & 0.252 & 50 \\
\hline
\end{tabular}

* The range of uncertainty in the data is indicated by the 4 per cent variation in $l_{\mathrm{d}}$ for the two $1.94-\mathrm{mM}$ uridine solutions. There is considerable error in the slope values $\left(E_{1 / 4}-E_{3 / 4}\right)$ due to the noise in the $E_{3 / 4}$ region of the wave.

$+I_{\mathrm{d}}=i_{\mathrm{d}} / \mathrm{Cm}^{2 / 3} t^{\mathrm{i} / 6}$

$\left(\mu \mathrm{A} \mathrm{s}^{1 / 2} \mathrm{mM}^{-1} \mathrm{mg}^{-2 / 3}\right)$.

\# An equivalent amount of hydroxide ion was present; an anodic wave was produced.

decrease in the fraction of RH serving as proton donor with increasing RH concentration would be inconsistent with results for the other hydroxypyrimidines studied[1-3]. A more likely cause is an ECEC mechanism, similar to that for thymine [3], in which $\mathbf{R H}_{2}$ is reduced and protonated to form $\mathrm{RH}_{3}$. The likelihood of such an ECEC mechanism is supported by cyclic voltammetric data ( $c f$. subsequent discussion). The possible nature of the overall reduction mechanism is discussed in the subsequent section on Mechanism.

Phase-selective ac polarography. Uridine $(0.2$ to $2.0 \mathrm{mM}$ ) was examined at 40,100 and $150 \mathrm{~Hz}$. No quadrature current component peak was observed except at ca. $-2.3 \mathrm{~V}$ for a $1.90-\mathrm{mM}$ uridine solution at $40 \mathrm{~Hz}$ (Fig. 2). This peak may reflect adsorption of uridine at high concentration with a resulting desorption on reduction (cf. previous reference $[10,11]$ to adsorption of uridine from aqueous medium).

The in-phase current component (Table $2 ; \mathrm{Fig}$. 2 ) is distinguished by the asymmetry of the peak, which apparently largely reflects the reversible reduction of uridine on the more positive potential side and the follow-up reactions (eg, father-son) on the more negative potential side.

Cyclic voltammetry. Cyclic voltammetry at a scan rate, $v$, of 0.03 to $0.2 \mathrm{~V} \mathrm{~s}^{-1}$ shows a cathodic peak at about $-2.3 \mathrm{~V}$ for the le reduction of uridine to the radical anion. Since no complementary anodic peak is seen at up to $v$ of $239 \mathrm{~V} \mathrm{~s}^{-1}$, either the uridine reduction is very irreversible, which is unlikely, or the reduction product is rapidly consumed in a chemical reaction. 2-HP, uracil and thymine, which are involved in follow-up father-son processes, exhibit similar behavior $[1-3]$.

At $v$ less than about $0.24 \mathrm{Vs} \mathrm{s}^{-1}$, cathodic and anodic peaks appear at $-0.25 \mathrm{~V}$ and can be attributed to the redox couple,

$$
\mathbf{R} \mathbf{H g}+e \rightleftharpoons \mathbf{R}^{-}+\mathbf{H g} \text {. }
$$

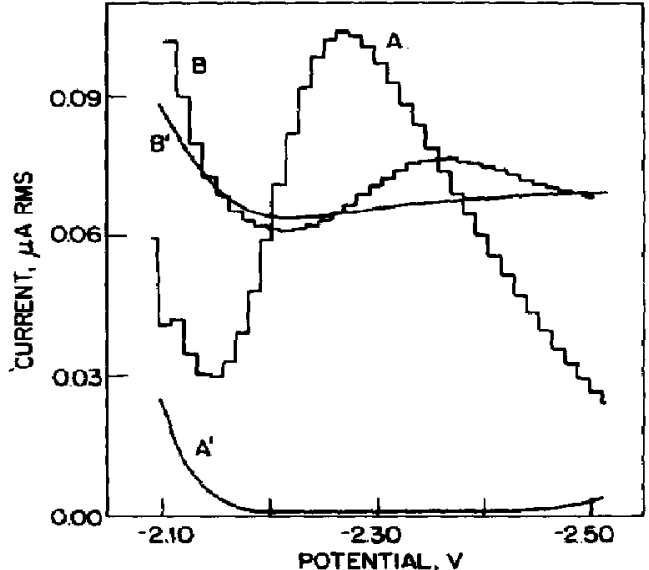

Fig. 2. ac polarogram of $1.90 \mathrm{mM}$ uridine in DMSO (0.1 M TBAP). Alternating voltage $=10 \mathrm{mV}$ peak-to-peak; frequency $=40 \mathrm{~Hz}$. A: in-phase current component; B: quadrature current component; $A^{\prime}$ and $B^{\prime}$ : respective current components for background solution alone.

Table 2. Characteristics of the ac polarographic in-phase current component of uridine in DMSO (0.1 M TBAP)

\begin{tabular}{lccccc}
\hline $\begin{array}{c}\text { Concn } \\
(\mathrm{mM})\end{array}$ & $\begin{array}{c}-E_{\mathrm{s}} \\
(\mathrm{V})\end{array}$ & $\begin{array}{c}\mathrm{i}_{\mathrm{s}} \\
(\mu \mathrm{A}, \mathrm{rms})\end{array}$ & $\begin{array}{c}\mathrm{f} \\
(\mathrm{Hz})\end{array}$ & $\begin{array}{c}E_{+8 / 2}^{*}-E_{\mathrm{s}} \\
(\mathrm{V})\end{array}$ & $\begin{array}{c}E_{\mathrm{s}}-E_{-\mathrm{s} / 2}^{\dagger} \\
(\mathrm{V})\end{array}$ \\
\hline 0.190 & 2.27 & 0.018 & 40 & 0.08 & 0.11 \\
0.190 & 2.29 & 0.026 & 100 & $\ddagger$ & 0.17 \\
0.190 & 2.29 & 0.035 & 150 & $\vdots$ & $>0.18$ \\
1.90 & 2.27 & 0.104 & 40 & 0.07 & 0.17 \\
1.90 & 2.29 & 0.114 & 100 & 0.10 & 0.16 \\
1.90 & 2.27 & 0.123 & 150 & 0.10 & 0.18 \\
\hline
\end{tabular}

- $E_{+s / 2}$ is the potential on the positive side of the peak where the current is $0.5 i_{s}$.

$+E_{-s / 2}$ is the potential on the negative side of the peak where the current is $0.5 i_{s}$.

\# The peak is not sufficiently resolved from the impurity peak at $-2.0 \mathrm{~V}$ to determine $E_{+\mathrm{s} / 2}$.

At higher scan rates, the peak pattern due to this couple can not be resolved from an impurity peak. Uracil behaves similarly except that two such anodic peaks can be seen at low $D(2)$

Variation of the cyclic voltammetric behavior of $0.55 \mathrm{mM}$ uridine with $v$ is summarized in Table $3 ; d c$ polarographic data for this solution are given in Table 1. The apparently lincar $i_{p}-v^{1 / 2}$ relation (Fig. 3) suggests either that the reduction is diffusion controlled at this concentration or that a totally irreversible kinetically controlled process is involved. However, examination of the Table 3 data indicates the nonlinear nature of the $i_{p}-v^{1 / 2}$ relation, if a zero intercept is assumed; the apparent linearity of Fig. 3 is due to insensitivity of the plot to data at low $i_{p}$ and $v^{1 / 2}$ values.

The linear shift of $E_{\mathrm{p}}$ to more negative potential with increasing scan rate (plotted as $\log v$ ) (Fig. 4) is as expected for a reversible electron-transfer reaction followed by a rapid chemical reaction where the effect of the latter is diminished as $v$ increases. The theory for a totally irreversible electron-transfer mechanism, for 
Table 3. Cyclic voltammetric reduction of $0.455 \mathrm{mM}$ uridine in DMSO (0.1 M TBAP)

\begin{tabular}{llll}
$\begin{array}{c}v \\
\left(\mathrm{~V} \mathrm{~s}^{-1}\right)\end{array}$ & $\begin{array}{c}E_{\mathrm{p}} \\
(\mathrm{V})\end{array}$ & $\begin{array}{c}i_{\mathrm{p}} \\
(\mu \mathrm{A})\end{array}$ & $\begin{array}{c}i_{\mathrm{p}} / A c v^{1 / 2 *} \\
\left(\mu \mathrm{As}^{1 / 2} \mathrm{~cm}^{-2}\right. \\
\left.\mathrm{mM}^{-1} \mathrm{~V}^{-1 / 2}\right)\end{array}$ \\
\hline 0.0312 & -2.27 & 0.32 & 290 \\
0.234 & -2.36 & 0.80 & 270 \\
23.9 & -2.42 & 5.5 & 180 \\
59.8 & -2.49 & 10 & 210 \\
149 & -2.52 & 16 & 210 \\
239 & -2.56 & 20 & 210 \\
\hline$*$ Electrode & area $(A)=0.0316 \mathrm{~cm}^{2} ;$ concentration $(c)$ \\
$=0.455$ mM. &
\end{tabular}

which the irreversibility is induced by a rapid chemical reaction, ie, a reversible electron-transfer followed by an irreversible, first-order chemical reaction, predicts a linear variation of $E_{\mathrm{p}}$ with $\log v$ with $\mathrm{d} E_{\mathrm{p}} / \mathrm{d}(\log v)$ equal to $-30 \mathrm{mV} /$ decade[15]). The observed slope of $\mathrm{ca}$ $-50 \mathrm{mV} /$ decade could be due to a quasireversible electron-transfer step with $\alpha \mathbf{0 . 5}$ and/or the effects of the father-son reaction.

The peak heights for a $1.94 \mathrm{mM}$ uridine solution (Fig. 5; Table 4) are dependent on the positive switching potential, $E_{\lambda}$. (Because of uncertainty in the electrode areas and possible variation of the current function with concentration, these peak heights are reported in arbitrary units with Figs $5 \mathrm{~A}$ and $5 \mathrm{~B}$ being comparable and Figs $6 \mathrm{~A}$ and $6 \mathrm{~B}$ being comparable; the scans were continuous and were recorded after a stcady state was attained.) When $E_{\lambda}$ is increased from -0.11 to $+0.18 \mathrm{~V}$, the peak Ic height (due to uridine reduction) increases from 0.7 to 1.6 units, and peaks

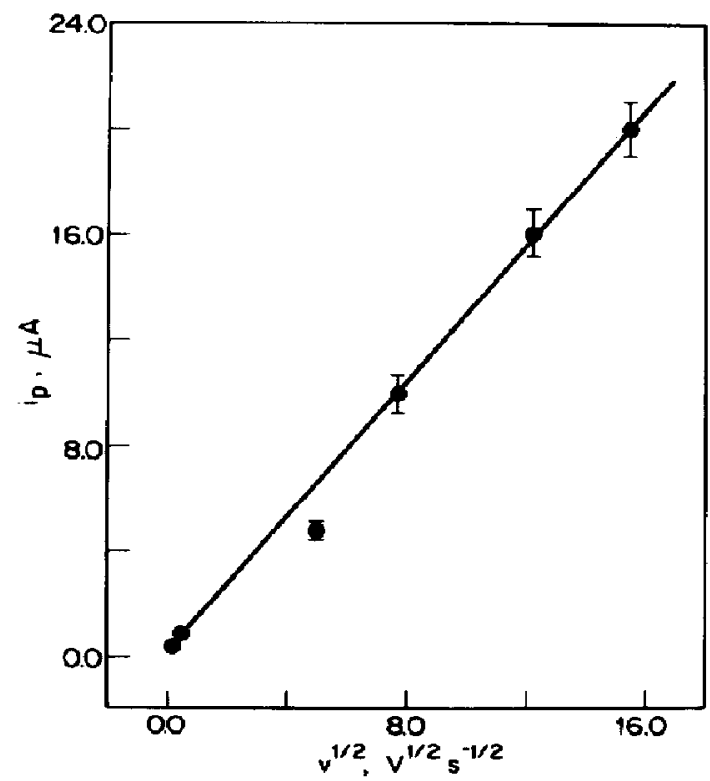

Fig. 3. Variation of the cyclic voltammetric peak current $\left(i_{p}\right)$ with the square root of the scan rate (v) for the reduction of $0.455 \mathrm{mM}$ uridine in DMSO (0.1 M TBAP). A single sawtooth potential function was initiated at $-1.5 \mathrm{~V}$ in the negative direction. Currents are indicated with $\pm 5 \%$ uncertainty.

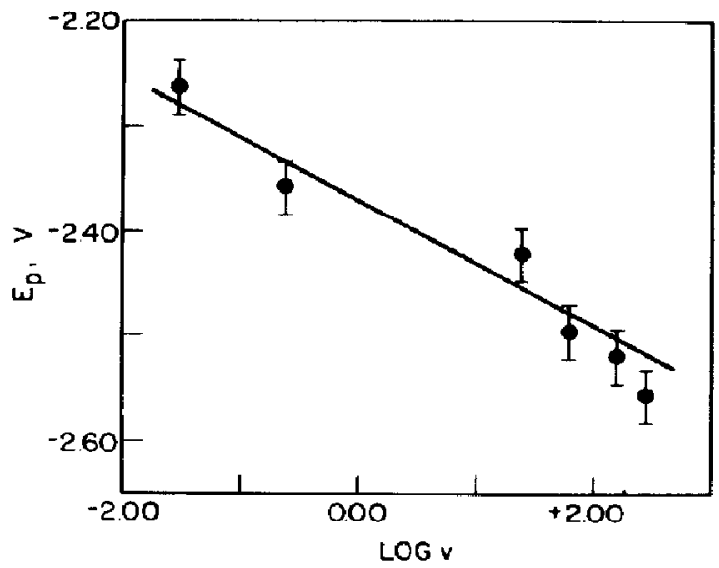

Fig. 4. Variation of the cyclic voltammetric peak potential $\left(E_{p}\right)$ with $\log$ of the scan rate $(v)$ in $\mathrm{V} \mathrm{s}^{-1}$ for the reduction of $0.455 \mathrm{mM}$ uridine in DMSO (0.1 M TBAP). A single sawtooth potential function was initiated at $-1.5 \mathrm{~V}$ in the negative direction. Potentials are indicated with an uncertainty of $\pm 20 \mathrm{mV}$.

IIa and II (assigned to the mercury-uridine anion couple) decrease from 2.3 and 2.0 to 1.2 and 1.5 units, respectively. Evidently an electrochemical process occurring at a potential more positive than $-0.11 \mathrm{~V}$ generates uridine, which is then available for reduction at $-2.45 \mathrm{~V}$, apparently at the expense of the peak II couple. Peak I la may correspond not only to oxidation of $\mathrm{H} \mathrm{g}$ in the presence of uridine anion ( $\left.\mathrm{R}^{-}\right)$(4) but also to other oxidation processes, eg, oxidation of $\mathrm{Hg}$ due to presence of the uridine dimer anion, $\left(\mathrm{RH}_{3}^{2-}\right.$ and trace impurities such as chloride ion. The sharp edge on the negative potential side of peak $11 \mathrm{cmay}$ be due to stripping of a $\mathrm{HgR}$ or other mercury salt film from the electrode surface.

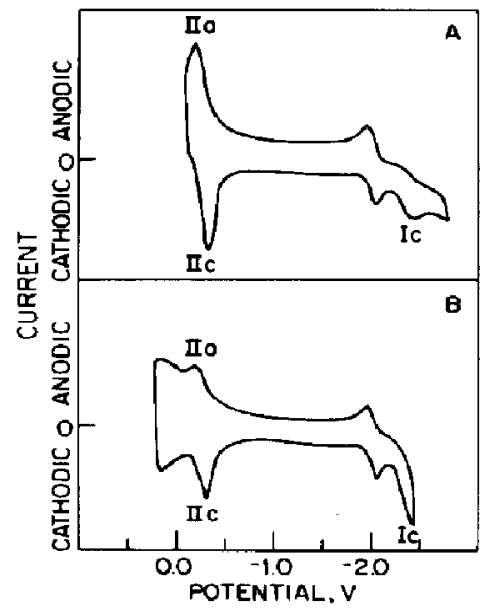

Fig. 5. Cyclic voltammograms of $1.94 \mathrm{mM}$ uridine in DMSO (0.1 M TRAP). Scan rate $=55 \mathrm{~V} \mathrm{~s}^{-1}$. A continuous sawtooth potential function was used; the first scan was initiated between -1.0 and $-1.9 \mathrm{~V}$ in the negative direction. Positive and negative switching potentials: $A,-0.11$ and $-2.85 \mathrm{~V}$, respectively; $B, 0.18$ and $-2.52 \mathrm{~V}$, respectively. 
Table 4. Characteristics of the cyclic voltammograms of $1.94 \mathrm{mM}$ uridine in DMSO (0.1 M TBAP)*

\begin{tabular}{|c|c|c|c|c|c|c|c|c|c|c|}
\hline \multirow{3}{*}{ Figure } & \multirow{3}{*}{$(\mathrm{V} / \mathrm{s})$} & \multirow{3}{*}{$\begin{array}{c}\boldsymbol{E}_{d}^{+} \\
(\mathrm{V})\end{array}$} & \multirow{3}{*}{$\begin{array}{l}\mathbf{E}_{\mathbf{Q}}^{\ddagger} \\
(\mathbf{V})\end{array}$} & \multicolumn{7}{|c|}{ Peaks } \\
\hline & & & & \multicolumn{2}{|c|}{$\mathbf{I}_{\mathrm{c}}$} & \multicolumn{2}{|c|}{$\mathrm{II}_{\mathrm{c}}$} & \multicolumn{2}{|c|}{$\mathbf{I I}_{\mathbf{a}}$} & \multirow{2}{*}{$\frac{\text { III }_{\mathrm{c}}}{E_{\mathrm{p}}}$} \\
\hline & & & & $\boldsymbol{E}_{\mathrm{p}}^{r}$ & $i_{p}$ & $E_{\mathrm{p}}$ & $i_{p}$ & $E_{\mathrm{p}}$ & $i_{p}$ & \\
\hline $\begin{array}{l}5 A \\
5 B \\
6 A \$ \\
6 B \S\end{array}$ & $\begin{array}{l}55 \\
55 \\
\$ 2 \\
52\end{array}$ & $\begin{array}{r}-0.11 \\
+0.18 \\
+0.03 \\
+0.48\end{array}$ & $\begin{array}{l}-2.85 \\
-2.53 \\
-2.62 \\
-2.16\end{array}$ & $\begin{array}{l}-2.45 \\
-2.45\end{array}$ & $\begin{array}{l}0.7 \\
1.6\end{array}$ & $\begin{array}{r}-0.33 \\
-0.33 \\
-0.45 \\
-0.45\end{array}$ & $\begin{array}{l}2.0 \\
1.5 \\
1.6 \\
1.6\end{array}$ & $\begin{array}{l}-0.20 \\
-0.20 \\
-0.09 \\
-0.09\end{array}$ & $\begin{array}{l}2.3 \\
1.2 \\
1.7 \\
1.5\end{array}$ & $\begin{array}{l}-0.59 \\
-0.59\end{array}$ \\
\hline
\end{tabular}

* Peak currents are given in arbitrary units; Fig. $5 A$ is comparable to $5 \mathrm{~B}$; Fig. $6 \mathrm{~A}$ is comparable to 6B. $E_{\mathrm{p}}$ is in volts.

$t_{\lambda}$ is the posilive switching potential.

$E_{\Omega}$ is the negative switching potential.

\& An equivalent amount of hydroxide was present.

Since a proton source is necessary for regeneration of RH from $\mathbf{R}^{-}$and since solvent and background electrolyte are effectively aprotic, source of the regenerated RH must be a uridine dimer, some other RH-containing compound, and/or another species capable of proton donation. A possible reaction, which could account for the observed behavior, is oxidation at more positive potential of $\mathrm{Hg}$ to $\mathrm{Hg}(\mathrm{I})$ which replaces protons in the neutral uridine dimer (RH-RH); the liberated protons combine with uridine anion ( $R^{-}$) formed on reduction of $\mathrm{HgR}$ to simultaneously (a) increase the steady state RH concentration near the electrode, thus favoring growth of peak Ic, and (b) decrease the corresponding $\mathbf{R}^{-}$concentration, resulting in attenuation of the peak II couple. Previous studies [1-3] of the 2-hydroxypyrimidines indicate that $(\mathrm{RH})_{2}^{2-}$ does not remove $\mathrm{H}^{+}$from $\mathrm{RH}$, ie, there is no need to posiulate such á reaction; consequently, if $\mathrm{H}^{+}$is made available in the presence of both $\mathrm{R}^{-}$and $(\mathrm{RH})_{2}^{2-}$, the $\mathrm{H}^{+}$will combine with the $\mathbf{R}^{-}$.

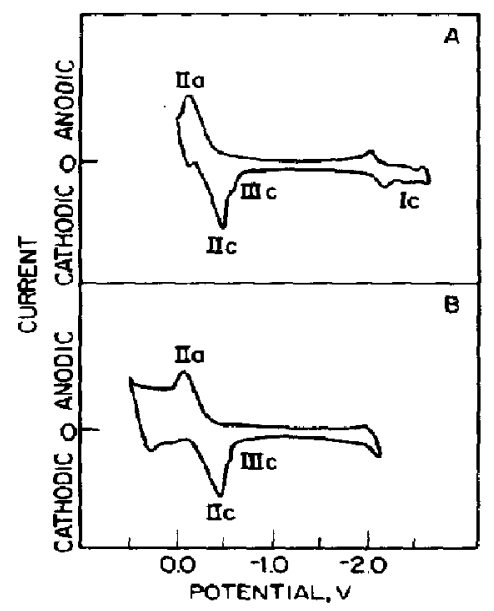

Fig. 6. Cyclic voltammograms of $1.94 \mathrm{mM}$ uridine in DMSO (O.1 M TBAP) in presence of an equivalent amount of hydroxide ion. Scan rate $=52 \mathrm{~V} \mathrm{~s}^{-1}$. A continuous sawtooth potential function was used; the first scan was initiated between -1.0 and $-1.5 \mathrm{~V}$ in the negative direction. Positive and negative switching potentials: $A, 0.03$ and $-2.62 \mathrm{~V}$, respectively; $B, 0.48$ and $-2.16 \mathrm{~V}$, respectively.
Another possible source of regenerated RH molecules is disproportionation of the radical anion into the parent compound and dianion, ie,

$$
2 \dot{\mathbf{R}} \mathbf{H}^{-} \rightleftharpoons \mathbf{R H}+\mathbf{R H}^{2-}
$$

The energetics of such reactions have been considered by Hush and Blackledge[12]; recently, Jaworski and Kalinowski[13] described an example of such a reaction in DMSO involving the disproportionation of 1 , 4-naphthosemiquinone radical anions. If such a process were occurring in the present study and if $\mathrm{Hg}^{+}$ affects the disproportionation equilibrium of uridine in the same manner as $\mathrm{Na}^{+}$and $\mathrm{K}^{+}$affect that of $1,4-$ naphthosemiquinone[13], ie, the $\mathrm{Hg}^{+}$generated at potentials positive of $-0.11 \mathrm{~V}$ combines with the uridine dianion and shifts the equilibrium of reaction 6 to the right, then RH would be regenerated when $E_{\lambda}$ is increased from -0.11 to $+0.18 \mathrm{~V}$.

Disproportionation of the type indicated by (6) would be favored by an increase in $\dot{R} H^{-}$concentration, $i e$, in original bulk solution uridine concentration, and would result in increases in observed reduction current and apparent faradaic $n$ due to the regeneration of $\mathbf{R H}$ in a repeating reaction sequence. Such behavior may contribute to the observed increase in the diffusion current constant with increasing uridine concentration (Table 1).

On addition of an equivalent molar quantity of hydroxide ion to the $1.94 \mathrm{mM}$ uridine solution (Fig. 6), peak Ic essentially disappears, IIIc appears at $-0.59 \mathrm{~V}$ as a small shoulder on II $c$, and IIa and IIc increase in height (relative to the respective charging currents of Figs 5 and 6); the behavior of peaks Ic and IIa-IIc is in accord with the added $\mathrm{OH}^{-}$deprotonating $\mathrm{RH}$ to generate an equivalent amount of $R^{-}$. The behavior on shifting $E_{2}$ to more positive potential is consistent with the absence of uridine dimers (neutral and anionic), ie, the change in peak II heights is within experimental error.

Peak IIIc may represent the reduction of a mercury hydroxide formed by residual unreacted hydroxide ion with formation of the compound (oxidation of mercury) being part of peak Ila. When $\mathrm{OH}^{-}$was added to DMSO, cyclic voltammetry produced anodic peaks at $-0.09 \mathrm{~V}$ and, for large amounts of $\mathrm{OH}^{-}$, at $-0.6 \mathrm{~V}$, and a possible complementary cathodic peak at $-0.2 \mathrm{~V}[1]$; these were attributed to the $\mathrm{Hg}(\mathrm{I})-\mathrm{Hg}(\mathrm{O})$ couple in the presence of an anion $\left(\mathrm{OH}^{-}\right)$which forms an insoluble compound with $\mathrm{Hg}(\mathbf{1})$. 
Impurity peaks at ca. $-2.0 \mathrm{~V}$, which may be due to the $\mathrm{Na}(\mathrm{I})-\mathrm{Na}(\mathrm{O})$ couple, decrease by a factor of 0.7 to 0.8 in the presence of uridine on cyclic voltammetry and $d c$ polarography; the cause for the decrease is not immediately apparent; analogous behavior was seen with 2-HP[1]. Anodic peaks due to oxidation of background elect rolyte and/or solvent impurity reduction products appear at slow scan rates $\left(v<0.23 \mathrm{~V} \mathrm{~s}^{-1}\right)$ when the switching potential, $E_{\lambda}$, is more negative than $c a$. $-2.6 \mathrm{~V}$.

\section{REACTION PATH}

The comparative $E_{1 / 2}$ values in DMSO obtained at $0 \mathrm{mM}$ (based on extrapolation of the data at low concentrations) and at $2 \mathrm{mM}$, respectively are -2.34 and $-2.31 \mathrm{~V}$ for uracil, and -2.20 and $-2.22 \mathrm{~V}$ for uridine. The appreciably easier electrochemical reduction of uridine than that of uracil can be ascribed to the electron-withdra wing effect of the ribose moiety. Thus, in aqueous media at $\mathrm{pH} 4.1, E_{1 / 2}$ is $-1.43 \mathrm{~V}$ for cytosine and $-1.38 \mathrm{~V}$ for cytidine (the corresponding cytosine nucleoside) [14].

The $d c$ polarographic data for the uridine reduction, $e g$, the concentration-dependencies of both $E_{1 / 2}$ and $I_{d}$, support the presence of one or more rapid chemical reactions following electron transfer. The behavior of the uridine furthermore supports its undergoing a post-electron-transfer father-son reaction, as well as the expected dimerizations, generally similar to those for the electrochemical behavior of $2-\mathrm{HP}$, uracil and thymine in DMSO [1-3]. The absence even at high scan rate of an anodic cyclic voltammetric peak complementing the uridine reduction peak further supports a reaction rapidly consuming the radical anion, which is the initial reduction product.

The $I_{\mathrm{d}}-c$ variation, using an $I_{\mathrm{d}}$ of 0.93 for a $1 e$ transfer, supports a concentration-dependent reduction mechanism, which seemingly varies from $1 e / 2$ uridine at infinite dilution to $2 e / 3$ uridine at $0.5 \mathrm{mM}$ uridine and $4 e / 5$ uridine at $2 \mathrm{mM}$ uridine; in view of the uncertainties in current measurement, these numbers are to be regarded as approximations which, as subsequently discussed, have an internal consistency.

A mechanism consistent with the foregoing and the other da ta is shown in Fig. 7. The uridine anion, as well as other species involved can be depicted in resonance forms, as shown below.

Although the pyrimidine bases, including uracil, have been postulated to be reduced initially at $C(4)[1-3,16], C(2)$ in uracil is more electron deficient than $C(4)$ (cf.[2] and citations therein). The ribose moiety on $\mathrm{N}$ (1) in uridine should increase the electrondeficiency at $C(2)$ relative to $C(4)$ by inductive electronwithdrawing effects; additionally, the proton of the ribose 5'-hydroxy group is suitably located for

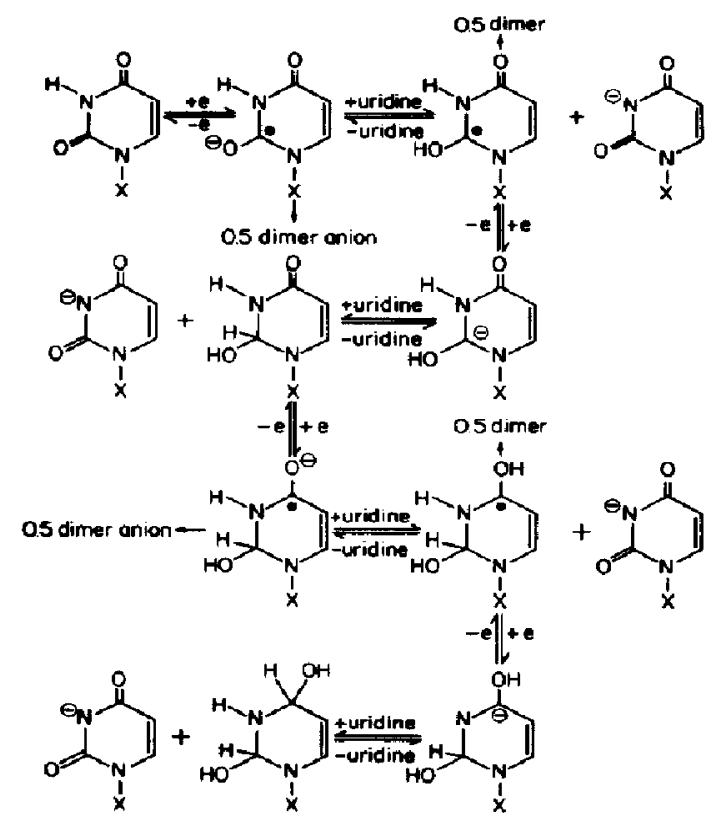

Fig. 7. Suggested reaction paths for the reduction of uridine in aprotic medium (DMSO), where $X$ represents the ribose group.

hydrogen-bonding to $O(2)$, which would further increase the eloctron-deficiency at $\mathbf{C}(2)$. Possible initial $l e$ injection at $\mathrm{C}(2)$ on uridine is supported by the apparently significantly more rapid protonation of the initially formed radical anion, as compared to uracil[2] and thymine [3], and the slower dimerization of the free radical thus formed, as compared to uracil[2]

In the case of uracil[2], the free radical dimerization is sufficiently rapid that little or none of the free radical is reduced. In thymine, the 5-methyl group apparently sterically hinders dimerization at $\mathrm{C}(4)$ of the free radical, thereby permitting its reduction. The ribose moiety in uridine is sufficiently far removed from C(4), so that it should not significantly sterically hinder dimerization at $\mathrm{C}(4)$; however, if initial $1 e$ reduction followed by the father-son reaction yields a free radical with the unpaired electron residing at $C(2)$, the ribose group at $\mathrm{N}(1)$ would offer considerable hindrance to dimerization and, hence, would facilitate further reduction.

Hydrogen-bonding between the 5'-hydroxy group and $O(2)$ would facilitate rapid intramolecular protonation of $O(2)$ in the radical anion formed on initial le reduction at $\mathrm{C}(2)$; intermolecular protonation of the resulting axygen anion on $\mathrm{C}\left(5^{\prime}\right)$ might be more rapid than protonation of the exocyclic oxygens on pyri-

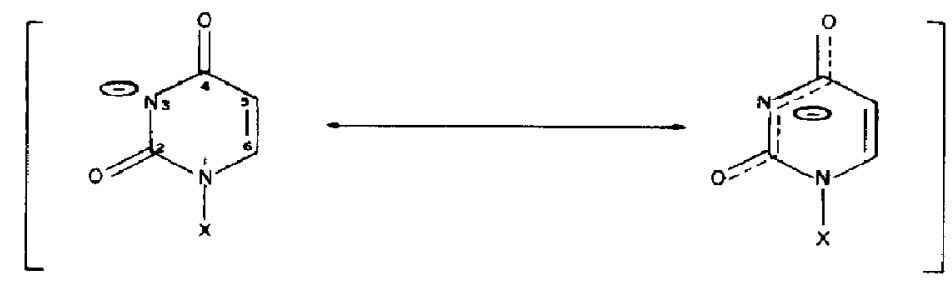


midine base radical anions. Thus, the father-son reaction at low uridine concentration could be sufficiently rapid to effect protonation of all of the radical anion formed on initial le reduction, resulting in an $n$ of 0.50 .

Since the free radical should be more easily reduced than uridine itself, $n$ would be expected to be either unity for an ECE process, ie, $2 e / 2$ uridine, or 0.67 for an ECEC process, ie, $2 e / 3$ uridine. The observed $n$ of 0.57 at $0.19 \mathrm{mM}$ is intermediate between those expected for an $\mathrm{EC}(0.50)$ and an ECEC (0.67) process, suggesting that some of the free radical is not reduced but is lost by dimerization. At such low concentration, the bimolecular father-son reaction may be sufficiently slow that some of the radical anion diffuses away from the electrode before being protonated; some of the free radical thus formed would be expected to dimerize before it could diffuse back to the electrode and be reduced; hence, an $n$ intermediate between those for EC and ECEC (or ECE) processes would be observed. As the uridine concentration increases, the rate of radical anion protonation should increase; hence, the distance that the radical anion must diffuse from the electrode before protonation (and, therefore, the distance that the free radical must diffuse back to the electrode surface) decreases and a larger fraction of it is further reduced.

At high concentration, $e g, 1.94 \mathrm{mM}$, the $n$ of 0.8 corresponds to addition of four electrons and four protons to a uridine molecule with concommitant formation of four uridine conjugate base molecules. Thus, at high concentration, protonation of the radical anion formed after addition of the third electron and reduction of the resulting free radical are sufficiently rapid that none of the free radical dimerizes. Whether addition of the third and fourth electrons involve reduction of the second carbonyl group or $C(5)=C(6)$ is not clear; however, in uracil, [2], electron densities on $C(5)$ and $C(6)$ are greater than on either $C(2)$ or $C(4)$.

The dependency of $n$ on uridine concentration did not allow unequivocal examination of the mechanism outlined in Fig. 7 by controlled electrode potential electrolysis of uridine, ie, the steadily decreasing uridine concentration on exhaustive electrolytic reduction would result in a constantly changing effective laradaic $n$ with concommitant change in product popalation.

Protonation source in uracil. Since uracil can be deprotonated at $\mathbf{N}(1)$ or $\mathrm{N}(3)$, when involved in a father-son reaction, $E_{1 / 2}$ for the uridine anion-mercury couple may be used to identify the two oxidation processes observed on adding a strong base to a uracil solution. These processes might be due to oxidation of $\mathrm{Hg}$ to $\mathrm{Hg}(\mathrm{I})$ involving salts formed at $\mathbf{N}$ (1) and $N(3)$ of the uracil with the $H g(1)$ ion [2], ie, the $d c$ polarogram for uracil with TEAH present exhibits an anodic wave with $E_{1 / 2}$ of $-0.25 \mathrm{~V}$ and the cyclic voltammogram $(v=0.106 \mathrm{~V} / \mathrm{s})$ shows anodic peaks at about $-0.25 \mathrm{~V}$ (peak Ia) and $+0.05 \mathrm{~V}$ (peak IIa). Uridine, which is protonated only at $N(3)$, produces on TEAH addition only a single anodic wave at $-0.25 \mathrm{~V}$ and a single anodic peak at $-0.25 \mathrm{~V}$ at slow scan rates. Assuming that the peak potentials at low scan rate are not markedly different from the $E_{1 / 2}$ and that the effect of the ribose on the salt formation is negligible, it is possible to assign uracil peak Ia as due to oxidation to a uracil salt bonded to $\mathrm{Hg}(\mathrm{I})$ at $\mathrm{N}(3)$ and peak I la due to the corresponding $N(1)$ salt.

Acknowledgement - The authors thank the National Science Foundation, which helped support the work described.

\section{REFEREN CES}

1. T. Wasa and P. J. Elving, J. electroanal, Chem. 91, 249 (1978).

2. T. E. Cummings and P. J. Elving, J. electroanal. Chem.94, 123 (1978).

3. T. E. Cummings and P. J. Elving, $J$. electroanal. Chem., 102, 237 (1979).

4. P. J. Elving, Can J. Chem. 55, 3392 (1977)

5. A. L. Lehninger, Biachemistry, 2nd edn p. 316 Worth, New York (1975).

6. T. E. Cummings, M. A. Jensen and P. J. Elving, Electrochint. Acta 23, 1173 (1978).

7. Y. Kondo, F. Yochizaki and T. Takemoto, Heterocycles 3. 163 (1975).

8. P. Cerutti, Y. Kondo, W. R. Landis and B. Witkop, J. Am. Chem. Soc. 90, 771 (1968).

9. E. Fahr, G. Fuerst, G. Doerhoefer and H. Popp. Agnew. Chem. Int. Ed. Engl. 6, 250 (1967).

10. V. Vetterl, J. electroanal. Chem. 19, 169 (1968).

11. V. Vetterl, Biophysik 5, 255 (1968).

12. N. S. Hush and J. Blackledge, J. Chem. Phys. 23, 514 (1955).

13. J. S. Jaworski and M. K. Kalinowski, Polish J. Chem. 52, 2019 (1978)

14. J. W. Webb, B. Janik and P. J. Elving, J. Am. Chem. Soc. 95, 991 (1973).

15. R. S. Nicholson and I. Shain, Analyt. Chem. 36, 706 (1964).

16. P. J. Elving, in Topics in Bioelectrochemistry and Bioenergetics, Vol. I, (Edited by G. Milazzo), pp. 179-286. John Wiley, London (1976). 